\title{
Looking for a Fit for Purpose: Business Process Maturity Models from a User's Perspective
}

\author{
Amy Van Looy ${ }^{1,2}$ \\ ${ }^{1}$ University College Ghent, Faculty of Business Administration \& Public Administration, \\ Department of Management \& ICT, Ghent, Belgium \\ amy. vanlooy@hogent. be \\ ${ }^{2}$ Ghent University, Faculty of Economics \& Business Administration, \\ Department of Management Information Science \& Operations Management, Ghent, Belgium \\ Amy . VanLooy@UGent. be
}

\begin{abstract}
Many books and articles have been written about the importance of business process management. However, in practice, improving business processes remains challenging. Therefore, business process maturity models (BPMMs) have been developed to assist organizations in their search for business process excellence (e.g. CMMI or OMG-BPMM). However, given their importance, a BPMM proliferation exists and practitioners are left without overview. Moreover, BPMMs differ in approach. An organization wishing to start with a BPMM thus risks selecting a model that does not fully fits its needs. The BPMM literature and the general literature on maturity models are mainly restricted to the design perspective. To our knowledge, the user's perspective is largely neglected. Based on an international Delphi study, this article introduces 14 criteria that (potential) BPMM users must consider to obtain a fit for purpose. The findings will be used to build a decision tool for BPMM selection.
\end{abstract}

Keywords: business process maturity, maturity model, selection, decision criteria, design research.

\section{$1 \quad$ Introduction}

Two paradigms dominate the research on business processes [1], and information systems (IS) [2,3]: (1) behavioral-science paradigm (i.e. building and testing theories to explain and predict situations), and (2) design-science paradigm (i.e. building and testing artefacts to solve problem situations). Research on BPMMs and maturity models in general is mostly situated in the second paradigm. For instance, the IS design research cycle has been translated towards maturity models [4,5]. It typically covers pre-design, design and post-design phases. Furthermore, Hevner et al. [2] formulate seven guidelines to evaluate the design of IS artefacts, which have also been translated towards maturity models [4]. The design-science paradigm thus examines maturity models as artefacts. March and Smith [3] distinguish four IS artefact types:

- construct: a conceptualization or vocabulary to describe problems and solutions;

- model: a description of problems and solutions, based on the conceptualization; 
- method: a set of steps (i.e. algorithms or guidelines) to perform a task;

- instantiation: the realization of constructs, models and methods into a tool.

Translated towards maturity models, Mettler and Rohner [5] confirm that a common conceptualization is lacking. Consequently, for this research, we describe a BPMM as ' a model to assess and/or to guide best practice improvements in organizational maturity and process capability, expressed in lifecycle levels, by taking into account an evolutionary road map regarding (1) process modeling, (2) process deployment, (3) process optimization, (4) process management, (5) the organizational culture, and/or (6) the organizational structure' [6, p.1132-1133]. A BPMM assesses (AS-IS) and improves (TO-BE) maturity. The latter is a collection of capabilities (e.g. skills or competences) that are needed for a business process to perform excellently. For instance, how capable is your organization to model its business processes in a graphical design, or to run them without errors? Maturity levels indicate the overall growth through all capabilities together, whereas capability levels indicate the growth per capability. The business process itself may consist of several sub processes, and defines how an organization operates. Furthermore, BPMMs are both models and methods, as they combine descriptions (e.g. maturity levels) with key practices (e.g. to achieve higher levels) [5]. Finally, instantiations can be documents or websites to assist organizations in using a BPMM.

To our knowledge, the BPMM literature is mainly restricted to a design perspective, i.e. by creating a design theory or by designing particular BPMMs, e.g. [7]. The research cycles, guidelines, and artefact types constitute a theory on the design of maturity models. When designed accordingly, BPMMs are supposed to have a sound methodological foundation. However, not all design criteria are relevant when choosing a BPMM. Moreover, also non-design criteria may come to the foreground during BPMM selection, such as financial or practical considerations. Consequently, this article takes a user's perspective. Particularly, we focus on the BPMM selection phase, which increases in importance as the number of BPMMs continues to increase. For instance, in the second quarter of 2010, we already collected a sample of 69 BPMMs regarding generic processes, supply chains and collaboration processes. We are of the opinion that investing in the BPMM selection phase pays off by saving money and efforts afterwards, i.e. for the corrective actions needed if a used BPMM turns out to be no fit for purpose.

Furthermore, the BPMM proliferation [8] raises questions about the substantial differences between BPMMs. Some comparative overviews have been made, albeit with a small number of BPMMs [9]. Mettler [10] also presents criteria for designing maturity models from a user's perspective, but not specific to the BPMM context and without an overview of existing models. On the other hand, Röglinger, Pöppelbuss and Becker [11] present a limited BPMM overview while proposing BPMM design criteria, but without practical advice on BPMM selection. Consequently, organizations have no comprehensive overview of academic and industry-owned BPMMs and an incomplete state of knowledge on how to select a BPMM that best fits their needs.

This article is a work-in-progress which elaborates on the decision criteria that (potential) BPMM users must consider when selecting a BPMM. It is based on a 
thorough methodological approach, explained in section 2. The criteria and their trade-offs are discussed in section 3, followed by the future research steps in section 4. Finally, the preliminary research findings are summarized in the conclusion section.

\section{Methodology}

The decision criteria for BPMM selection were obtained by consulting independent subject matter experts in an international Delphi study. A Delphi study is an established consensus-seeking decision-making method using 'a series of sequential questionnaires or rounds, interspersed by controlled feedback, that seek to gain the most reliable consensus of opinion of an expert panel' [12, p.458]. We have chosen a Delphi study as its iterative approach enhances validity, compared to a single questionnaire. Furthermore, according to Van De Ven and Delbecq [13], it generally results in a higher quantity and quality of ideas than other group decision-making methods. The experts are also anonymous, which minimizes group pressures. Moreover, a Delphi study is widely used for exploring ideas and structuring group communication on framework development and rating. Delphi examples are also present in IS research in general [14] and business processes in particular [7].

In November 2011, the Delphi study started with 22 BPM experts, i.e. 11 academics and 11 practitioners, each from five different continents. The academics had credible BPM(M) publications in academic journals, and the practitioners designed a BPMM, applied BPM(M), or were interested in BPMM selection. The selection procedure conforms to [14], introducing different backgrounds to minimize bias. Consensus conditions were a priori defined for a 7-point Likert scale, based on measures of location (i.e. frequencies) and spread (i.e. interquartile range) [15]: (1) 50\% of the experts must agree on the two most extreme scores (i.e. either 1-2 or 6-7), (2) $75 \%$ must agree on the three most extreme scores (i.e. either 1-2-3 or 5-6-7), (3) the interquartile range must be 1.50 or less, and (4) no opposite extreme score given by any expert (i.e. either 7 for the first case or 1 for the second). Per round, the responses were anonymously analyzed by four coders, of which one independent coder was from another university. This codification panel stopped iterating when the consensus conditions were met, or when results became repetitive. Hence, a Delphi study typically takes three to four rounds $[7,15]$.

After three Delphi rounds, consensus was reached for 14 decision criteria. Other criteria had no trend towards consensus due to condition 4, i.e. at least one expert with an opposite extreme score in multiple rounds. In all rounds, the response rates exceeded the minimum value of $70 \%$, enhancing research rigor and validity [15]. Moreover, $95 \%$ of the respondents in the third round $(\mathrm{N}=17)$ agreed that the set of final criteria is very to extremely important for BPMM selection (i.e. scores 6 or 7), with a median of 6 and an interquartile range of 0. In subsequent Delphi rounds, the experts were asked to determine which of these 14 decision criteria are more important in pairwise comparisons (i.e. Analytical Hierarchy Process). Besides ranking, it allows calculating weightings for assigning evaluation scores to existing BPMMs. As this article concerns a work-in-progress, the subsequent rounds are out-of-scope. 


\section{Preliminary Results}

We hereby present the criteria that a user must consider when selecting a BPMM. For reasons of comprehensiveness, they are grouped: (1) assessment criteria, i.e. how maturity is measured and by whom, (2) improvement criteria, i.e. what is measured as maturity, particularly the capabilities and their improvements to reach successive levels, and (3) non-design criteria, i.e. other criteria not directly related to assessment and improvement. Table 1 alphabetically shows the decision criteria per group.

Table 1. An alphabetical overview of the 14 decision criteria for BPMM selection

\begin{tabular}{l|l|l}
\hline Assessment criteria & Improvement criteria & Non-design criteria \\
\hline 1. Availability & 7. Architecture details & 12. Costs \\
2. Data collection & 8. Architecture type & 13. Purpose \\
3. Duration & 9. Capabilities & 14. Validation \\
4. Nr. of questions & 10. Nr. of processes & \\
5. Rating scale & 11. Type of processes & \\
6. Respondents & & \\
\hline
\end{tabular}

\subsection{Assessment Criteria}

Availability. Whether the assessment questions and corresponding level calculation are publicly available (instead of only known to the assessors). BPMMs do not always provide full details. This particularly counts for non-academic models, e.g. in consultancy. The user must decide whether this limited availability is an issue for the organization. For instance, fully known BPMMs (i.e. either free or charged) can be used for educating process team members or for earning credibility.

Data Collection. The way information is collected during an assessment. Objective techniques involve document reviews, and give an idea of how organizations work, without interrupting individuals or activities. They minimize biased results of (particularly internal) assessors and respondents. On the other hand, subjective techniques gather information about how organizations actually work, e.g. by questionnaires, interviews or observations. As it rather concerns personal beliefs, some precautions can be taken, e.g. a third party lead assessor, multiple assessors and respondents, data collection training, or a combination with objective techniques.

Duration. The maximal duration of a particular assessment. Some BPMMs only take one day (e.g. a quick scan within 15 minutes), whereas other BPMMs present a more profound analysis of one week or longer. As time is money, the user must consider how much time he wants to spent on the assessment alone.

Number of Questions. The maximal number of questions to be answered during an assessment. More questions provide more insight to develop a road map, but may be less feasible and/or take longer. Less than 20 questions are rather used as a teaser or a quick scan. 
Rating Scale. The type of data that is collected during an assessment. Quantitative data (i.e. discrete, interval or ratio scales) can be statistically analyzed and compared, independent of the assessors' interpretation. On the other hand, qualitative data (i.e. nominal or ordinal scales) provide more in-depth descriptions by delving into details. However, they depend more on the assessors' skills. Also a combination of rating scales is possible, depending on which data and skills are available.

Respondents. The explicit recognition to include people from outside the assessed organizations as respondents. If only internal respondents (i.e. managers and/or staff of the assessed organizations) are questioned, the user assumes that they fully know their stakeholders' needs. However, also involving stakeholders recognizes the need for an outside-in perspective by explicitly listening to stakeholders.

\subsection{Improvement Criteria}

Architecture Details. The degree of guidance that a BPMM gives on your journey towards higher maturity. It concerns the extent to which the road map (i.e. step-bystep plan) explains which criteria (i.e. goals and best practices) must be satisfied before reaching each particular level: (1) descriptive, (2) implicit prescriptive or (3) explicit prescriptive. A descriptive road map is limited to high-level descriptions. As it gives less support, it is suited for organizations wishing to become acquainted with BPMMs, or for organizations which are highly experienced with process improvements. An implicit prescriptive road map has criteria interwoven in the assessment questions, i.e. with an ordinal scale or a matrix, that explain all capabilities per level. Assessors can derive the criteria from the assessment questions. Finally, an explicit prescriptive road map gives most guidance by separately listing criteria from the assessment questions.

Architecture Type. The possibility to define a road map per capability, a road map for overall maturity, or both. It concerns linking (maturity of capability) levels to capabilities in a step-by-step plan, which explains how to reach each consecutive level. A continuous architecture provides capability levels per capability, i.e. one road map per capability. It allows organizations to assess and improve each capability separately, and thus to improve capabilities at a different pace or to limit their scope to only those capabilities they are interested in. As not all capabilities are necessarily taken into account, there is a risk for suboptimal optimizations (in terms of overall maturity). On the other hand, a staged architecture provides maturity levels linked to all capabilities together, i.e. one road map for overall maturity. The emphasis is on simultaneous advancements, instead of individual capability advancements.

Capabilities. The capabilities to be assessed and improved. BPMMs differ in the capabilities they actually address. They generally vary from basic capabilities related to the traditional business process lifecycle, i.e. modeling, deployment, optimization, and management, to the addition of organizational capabilities, i.e. to create a process-oriented culture and structure. In theory, all presented capabilities are required for fully mature business processes. However, in practice, an organization can 
opt for only a subset of capabilities, e.g. depending on the degree of top management support, IT background of the user, prior BPM experience, organization size, etc. For instance, organizations with local, bottom-up initiatives or with limited BPM experience might wish to start with the basic capabilities, limited to the traditional business process lifecycle. Additionally, the culture capability requires a minimum level of management support to promote business processes and granting (financial) rewards to process performance. Finally, structural configurations inherently require top management support. The latter is particularly recommended if you already have some BPM experience or if your ambition is to standardize processes across large departments or divisions. As capabilities are core to BPMMs, the user must select a set of capabilities that best fits its organizational needs.

Number of Processes. The number of business processes to be assessed and improved: (1) one, (2) more or (3) all. For BPMMs focusing on a single business process, the process boundaries must be defined by the user, e.g. is a business process assessed and improved as a sub process or as a separate process. BPMMs can also focus on more than one, but not all business processes within the assessed organizations. Assessment questions then deal with a particular business domain or value chain and all its (sub) processes. Furthermore, BPMMs can cope with all business processes in the assessed organizations. As such, assessment questions take a management perspective by focusing on how organizations deal with business processes in general, without focusing on particular processes. However, by improving the BPM mastery, it is likely that particular processes are indirectly improved too. Only few BPMMs combine specific processes (i.e. one or more) with the overall BPM mastery.

Type of Processes. Whether the BPMM is generic (i.e. for business processes in general) or domain-specific (e.g. for business processes in supply chains or collaboration situations). The terminology used in generic BPMMs, e.g. in the assessment questions, is likely to be more holistic. Benchmarking is possible across business domains. Accordingly, domain-specific BPMM use terminology adapted to their domain, which are likely to be less abstract to respondents and thus better understandable. However, benchmarking remains limited to organizations within the same domain. Hence, also this choice requires strategic considerations.

\subsection{Non-design Criteria}

Costs. The direct costs to access and use a BPMM. Not all BPMMs are free of charges. Particularly non-academic models may ask a one-off access fee or a required training to be followed. Recurring costs rather serve to pay a third party lead assessor, certification or benchmarking. The user must decide which budget can be spent, and adapt his expectations accordingly: you often get what you pay for. However, academic models can be free if they use your data for enhancing their research.

Purpose. The purpose for which a BPMM is intended to be used. The basic purpose of any BPMM is assessing and identifying process improvements, i.e. raising awareness. The key is recognizing deficiencies, creating willingness to act and to follow-through 
on the findings. Besides raising awareness, BPMMs can also allow benchmarking with other organizations (i.e. for comparing with competitors and sharing best practices) or certification (i.e. for external recognition of the assessment results, in line with the ISO quality certificates).

Validation. Whether or not empirical evidence is given that the BPMM helps to enhance the efficiency and effectiveness of business processes. Most BPMMs do not provide any proof of validity (or success). If they do, evidence is frequently limited to enumerating other organizations applying the model. Only few BPMM give evidence for the performance outcomes. The user must decide whether some proof of validity is required, depending on the planned investments. However, we strongly discourage the use of non-validated BPMMs. They can result in frustrations, time and money losses afterwards, i.e. if they appear to be flawed or unusable after you start using it.

\section{$4 \quad$ Future Work}

The decision criteria will be used to build a decision tool for BPMM selection, based on an online questionnaire and a decision table design. Particularly, by answering the questionnaire, the user will be guided to existing BPMMs that best fit his answers. Therefore, we will rely on a sample of 69 BPMMs. The final decision tool will be of practical use for organizations interested in assessing and improving their business processes, and for scholars who want to create or apply BPMMs. The next steps are:

1. Ranking and weighing decision criteria, and calculating evaluation scores for existing BPMMs.

2. Translating decision criteria and their options into a questionnaire.

3. Coupling the questionnaire to a decision table which comprises a mapping to existing BPMMs. BPMMs with low evaluation scores are omitted to guarantee quality.

4. Automating the questionnaire and the decision table in a proof-of-concept.

5. Conducting case studies. Managers will be asked to evaluate the tool and its output, i.e. by rating their satisfaction with the criteria and the proposed BPMMs.

\section{Conclusion}

This article discussed 14 criteria and their trade-offs that users must consider when selecting a BPMM, i.e. six assessment criteria, five improvement criteria, and three non-design criteria. They result from an international Delphi study with 22 BPM experts, both practitioners and academics from five continents, and will serve as input for a decision tool on BPMM selection. The novelty of this work-in-progress is that BPMMs are examined from a user's perspective, i.e. as a search for BPMMs that best fit the user's needs ('fit for purpose'). To our knowledge, it contrasts to current literature on maturity models which mainly takes a design perspective. Furthermore, the final decision tool will rely on a large sample of 69 BPMMs. At present, the criteria are alphabetically introduced. In future research, the experts will rank and weigh them to obtain a more refined overview and to grant evaluation score to existing BPMMs. 


\section{References}

1. Houy, C., Fettke, P., Loos, P.: Empirical Research in Business Process Management. Business Process Management Journal 16(4), 619-661 (2010)

2. Hevner, A.R., et al.: Design Science in Information Systems Research. MIS Quarterly 28(1), 75-105 (2004)

3. March, S.T., Smith, G.F.: Design and Natural Science Research on Information Technology. Decision Support Systems 15(4), 251-266 (1995)

4. Becker, J., Knackstedt, R., Pöppelbuss, J.: Developing Maturity Models for IT Management. Business \& Information Systems Engineering 1(3), 213-222 (2009)

5. Mettler, T., Rohner, P.: Situational Maturity Models as Instrumental Artifacts for Organizational Design. In: 4th DESRIST Conference, pp. 1-9. ACM, Malvern (2009)

6. Van Looy, A., De Backer, M., Poels, G.: Defining Business Process Maturity. A Journey towards Excellence. TQM \& Business Excellence 22(11), 1119-1137 (2011)

7. de Bruin, T., Rosemann, M.: Using the Delphi Technique to Identify BPM Capability Areas. In: 18th ACIS Conference, Toowoomba, pp. 642-653 (2007)

8. Sheard, S.A.: Evolution of the Frameworks Quagmire. IEEE Computer 34(7), 96-98 (2001)

9. Maier, A.M., Moultrie, J., Clarkson, P.J.: A Review of Maturity Grid based Approaches to Assessing Organizational Capabilities. In: Academy of Management Meeting (2008)

10. Mettler, T.: A Design Science Research Perspective on Maturity Models in Information Systems. Report BE IWI/HNE/03. Institute of Information Management, St. Gallen (2009)

11. Röglinger, M., Pöppelbuss, J., Becker, J.: Maturity Models in Business Process Management. Business Process Management Journal 18(2), 7 (2012)

12. Dalkey, N., Helmer, O.: An Experimental Application of the Delphi Method to the Use of Experts. Management Science 9(3), 458-467 (1963)

13. Van De Ven, A.H., Delbecq, A.L.: The Effectiveness of Nominal, Delphi, and Interacting Group Decision Making Processes. The Academy of Management Journal 17(4), 605-621 (1974)

14. Okoli, C., Pawlowski, S.D.: The Delphi Method as a Research Tool: an Example, Design Constructions and Applications. Information \& Management 42, 15-29 (2004)

15. Hasson, F., Keeney, S., McKenna, H.: Research Guidelines for the Delphi Survey Technique. Journal of Advanced Nursing 32(4), 1008-1015 (2000) 